colleagues in both recognizing and dealing with the impaired physician. More importantly, he discusses therapy for these individuals and indicates the chance of their returning to a functional practice of medicine.

\section{Environmental hazards}

This presentation is intended to convey a personal view of some of the environmental hazards associated with anaesthesia. The hazards include (a) obstetric problems, (b) liver and kidney disease and various cancers and (c) neurological deficits particularly those associated with nitrous oxide.

\section{Obstetric problems}

This is the most widely recognised environmental hazard of anaesthesia but individual opinions, as well as governmental and professional reactions, differ widely. Some feel that not enough is being done about the problem while others maintain either that there is no problem and that much research and finance has been misdirected, or that scavenging devices introduce an unacceptable hazard to the patient. In general many feel that this is a well worn subject about which there is considerable indifference and confusion amongst general operating room staff.

The epidemiological data has been reviewed before (e.g. Vessey and Nunn ${ }^{1}$ ). New information includes the recent Swedish survey (Axelsson and Rylander ${ }^{2}$ ) as well as the Finnish study of obstetrical hazards associated with other aspects of hospital work (Hemminki et al ${ }^{3}$ ).

The animal studies have two contributions to make to the solution of the problem. First, they provide evidence for a cause and effect relationship based on exposure to specific drugs. Second, where there is a satisfactory animal model, it may be possible to determine rational criteria for maximum "safe" levels of contamination. Good examples of the two objectives are provided respectively by the current studies with isofiurane (Mazze et $a l .^{4}$ ) and nitrous oxide (Sharer et al..$^{5}$ ).

\section{Non-obstetric risks}

The information on other health hazards is at the moment less than adequate to allow any firm conclusions. Most British anaesthetists are easily persuaded that disease of the lumbar spine is an occupational hazard associated with transferring patients in the operating room. However, there are serious limitations to the self-reporting of diseases. Various studies have implied that chronic exposure to anaesthetics is associated with a 1.6-1.7-fold increase in liver disease, and a 1.2-1.7-fold increase in kidney disease (Cohen et al. ${ }^{6}$ ) while a morbidity study of British doctors revealed no unusual pattern among anaesthetists compared with other specialists except in increased number of deaths from cancer of the pancreas (Doll and Peto ${ }^{7}$ ). It may be relevant to many occupational groups to note that approximately 50 per cent of pancreatic cancers are thought to be attributable to coffec drinking, a relative risk of 2.7 associated with three or more cups daily (MacMahon et al. ${ }^{8}$ ). A more objective assessment of morbidity would be provided by a study of early retirement from medical practice but it appears that the information required is not easily available.

\section{Neurological deficit associated with nitrous oxide} Nitrous oxide is the only inhalational anaesthetic which undergoes a conventional chemical reaction in vivo. It oxides the cobalt ion of vitamin $\mathrm{B}_{12}$ which results in inactivation of this cofactor in folate related metabolic pathways (Nunn and Chanarin $^{9}$ ). One of the consequences is a condition resembling subacute combined degeneration of the spinal cord, which has been reported in at least 15 people who had abused nitrous oxide (Layser ${ }^{10}$ ). This is probably related to methionine depletion following reduced activity of methionine synthase, the enzyme responsible for recycling methionine and which requires vitamin $B_{12}$ as a bound cofactor.

Methionine plays a crucial role in the synthesis of thymidine, one of the bases of deoxyribonucleic acid (DNA). This is almost certainly the cause of megaloblastic anaemia and leucopenia which follows prolonged administration and could also explain the fetotoxic effects of nitrous oxide associated with chronic exposure to low concentrations. Recent evidence suggests that these potential toxicities have a complex concentration and dose depen- 
dency relationship with an unexpectedly steep dose-response curve. The implications for the "safe level" of anaesthetic contamination have to be reexamined.

\section{References}

1 Vessey MP, Nunn JF. Occupational hazards of anaesthesia. Br Med J, 1980; 281: 696.

2 Axelsson $G$, Rylander $R$. Exposure to anaesthetic gases and spontaneous abortion: response bias in a postal questionnaire study. Int J Epidemiology 1982; 11: 250 .

3 Hemminki K, Mutanen P, Salontemi I, Niemi $M-L$, Vainio $H$. Spontaneous abortions in hospital staff engaged in sterilising instruments with chemical agents. Br Med J 1982; 285: 1461 .

4 Mazze RI, Wilson Al, Rice SA, Baden JM. Effects of isoflurane on reproduction and fetal development in mice. Anesth Analg 1984; 63: 249.

5 Sharer NM, Nunn JF, Royston JP. Chanarin 1. Effects of chranic exposure to nitrous oxide on methionine synthase activity. Br J Anaesth 1983; 55: 693.

6 Cohen EN, Brown BW, Wu ML et al. Occupational disease in dentistry and chronic exposure to trace anesthetic gases. J Am Dental Assoc 1980; 101: 21.

7 Doll $R$, Peto $R$. Mortality among doctors in different occupations. Br Med J 1977; 1: 1433.

8 MacMahon B. Yen S. Trichopoulos D et al. Coffee and cancer of the pancreas. N Eng J Med 1981; 304: 630 .

9 Nunn JF, Chanarin I. Nitrous oxide and vitamin $\mathrm{B}_{12} . \mathrm{Br} \mathrm{J}$ Anaesth 1978; 50: 1089

10 Layser RB. Myeloneuropathy after prolonged exposure to nitrous oxide. Lancet 1978; 2: 1227.

\section{Hepatitis, herpes and AIDS}

The recognition of occupational virus infections among anaesthetists is relatively recent. We have begun to recognize these potential hazards because of great advances in the clinical, epidemiologic and, particularly, the diagnostic approaches to these infections. This discussion will centre around two well known viral hazards for anaesthetists - hepatitis $B$ and herpes simplex virus. In addition, an update will be given of the current understanding of the transmission of the AIDS agent.

Hepatitis B virus is a DNA virus which cannot grow except in humans and selected simian primates. However, this virus can be associated with chronic human infections in individuals known as "carriers," who are often unaware of their potential infectiousness for others. The hazards to exposed susceptible individuals include the development of illnesses such as acute hepatitis, chronic hepatitis, cirrhosis, and hepatoma. The transmission of infectious virus is usually through unrecognized exposure to small amounts of infectious blood via mucous membranes or skin lesions, or by percutaneous mechanisms such as needlesticks. Seroepidemiologic studies have demonstrated a wide range in the prevalence of markers indicating previous exposure to hepatitis B virus, based on the individual's lifestyle, underlying disease, and occupation.

Physicians represent a group with a moderately elevated prevalence of serologic markers to hepatitis B virus. Anaesthetists have prevalence markers which indicate a risk similar to other medical workers who are frequently exposed to blood (approximately five to eight times that of the general population). A recent study in the United States has shown that the overall prevalence of all markers of hepatitis $B$ infection among 86 anaesthesia personnel was 23.3 per cent. A factor significantly associated with the presence of markers for hepatitis $B$ was the number of years of practice since graduation from medical school, presumably reflecting cumulative patient exposure. The country of origin of the anaesthetist has also been shown to be relevant in the prevalence of hepatitis $\mathbf{B}$ virus markers, therefore establishing this factor as a potentially confounding variable.

Attempts to avoid health care worker exposure to hepatitis $B$ virus by screening on the basis of historical information provided by the patient is unreliable. Routine screening of all patients for the hepatitis B carrier state is not justified, but serologic screening of high risk groups may be warranted to reduce the risk of exposure to personnel. The use of gloves and appropriate precautions with venipunctures and the handling of infected blood products should then be implemented. Current efforts at 